\title{
Comprehension of verb directionality in LIS and LSF
}

\author{
Valentina Aristodemo ${ }^{1}$, Beatrice Giustolisi ${ }^{2}$, Carlo Cecchetto ${ }^{2,3}$, Caterina \\ Donati $^{1}$ \\ ${ }^{1}$ Laboratoire Linguistique Formelle, Université de Paris, France \\ ${ }^{2}$ Department of Psychology, University of Milan - Bicocca, Italy \\ ${ }^{3}$ UMR 7023 Structures Formelles du Langage, Université Paris 8, France \\ https://doi.org/10.36505/ExLing-2020/11/0008/000423
}

\begin{abstract}
The present work reports the results of a comprehension task on verb directionality in Italian Sign Language (LIS) and French Sign Language (LSF) considering native and non-native signers. Our goals were to study age of first language exposure effects on the comprehension of verb agreement in LIS and LSF, to verify whether a significant difference between forward and backward directionality was found, and see if our results may provide insight about the nature (gestural vs. linguistic) of verb directionality in sign languages. In both languages we found that the ability to comprehend verb agreement is affected in non-native signers. This indicates that delayed first language exposure has long lasting effects in adulthood. We argue that our results support analyses of verb agreement as a fully grammatical phenomenon.
\end{abstract}

Keywords: agreement, directional verbs, sign language, age of exposure, Italian, French

\section{Introduction}

In sign languages (SLs), directional verbs move from the position in space associated to an argument towards the position associated to another argument. These verbs have been called 'agreeing verbs' by Padden (1988).

The interpretation to be given to of verb directionality in SLs has triggered much discussion and controversy among linguists. While some scholars use the term 'agreeing verbs' and insist that directionality is a fully grammatical phenomenon (Padden 1988, Pfau et al. 2018, a.o.), other scholars (e.g. Liddell 1995) deny that verb directionality can be assimilated to agreement and use a different terminology for these verbs, which are sometime called indicating verbs' or 'directional verbs' (Pfau et al. 2018 for an assessment of this literature) insisting on their gestural nature. For concreteness, in this paper we use the term 'agreeing verbs' to refer to these verbs.

Agreeing verbs can be further categorized into forwards and backwards verbs. Forwards verbs incorporate a movement going from the subject position to the object position, while backwards verbs show the inverse pattern, i.e. a movement from the object position to the subject position.

Behavioral studies have shown that comprehension of verb directionality is affected by age of first language exposure (AoE) in American Sign Language

ExLing 2020: Proceedings of $11^{\text {th }}$ International Conference of Experimental Linguistics, 12-14 October 2020, Athens, Greece 
(ASL) and British Sign Language (BSL) (Emmorey et al. 1995, Cormier et al. 2012, a.o.). AoE is a crucial variable to consider when assessing SLs comprehension: in fact, only a minority of deaf children are native signers, namely they acquire a SL from birth having at least a deaf signing parent. The majority of deaf children have hearing speaking parents, and if they are exposed to a SL, this typically occurs in kindergarten, or later.

In the present work, we investigated the comprehension of verb directionality in LIS and LSF. We collected data from 3 groups of signers: i) native signers (exposed to a SL from birth and with at least one signing parent), ii) early signers (AoE between 2 and 6 ), iii) late signers (AoE between 6 and 15). Our goals were: i) to evaluate whether AoE has an impact on directionality comprehension in LIS and LSF; iii) to verify if there is any difference in comprehension between forwards and backwards verbs; iii) to investigate whether effects of AoE can contribute to the debate about the nature of verb directionality.

\section{The present study}

Participants saw a brief non-linguistic clip showing three characters interacting, followed by a LIS/LSF sentence containing an agreeing verb, either forwards or backwards, and they had to judge whether the sentence matched the situation described in the clip. The sentence could either correctly describe the situation (match condition), or describe the situation attributing different thematic roles to the characters or displaying wrong argument selection (mismatch condition. Sentences were always signed by character A, who was therefore the grammatical first person, to character B (the grammatical second person). In the example, the matched sentence was "I yelled at you", whereas a mismatched sentence could be either "You yelled at me" (thematic roles inversion) or "I yelled at C" (wrong argument selection). Control sentences, which were clearly wrong, were added (like "I kissed you" in relation to the aforementioned example).

\section{Materials}

In LIS, the task included 18 situations and 4 sentences for each condition (1 correct, 2 with mismatched agreement, 1 control), for a total of 72 sentences. 12 forwards verbs and 6 backwards verbs were used. Participants saw all 72 sentences divided in two blocks.

In LSF, the task included 24 situations and 2 sentences for each situation (1 correct and 1 with mismatched agreement) and 20 control sentences, for a total of 68 sentences. 18 forwards verbs and 3 backwards verbs repeated twice were used. Participants saw the 68 sentences divided in two blocks, which were administered in two separate days.

In both tasks, items were pseudorandomized so that the same situation could not be repeated twice in a row. 


\section{Participants}

Forty-one Deaf LIS signers participated. Three participants were excluded because their score was below $75 \%$ accuracy in control sentences. The final sample consisted of 38 signers (mean age $=46 \mathrm{yrs}, \mathrm{sd}=9 \mathrm{yrs}$ ): 14 native, 13 early and 11 late.

As for LSF, 49 Deaf signers participated. Five participants were excluded because they were exposed to sign language when they were older than 15 . Four participants were excluded because their score was below $75 \%$ accuracy in control sentences. The final sample consisted of 40 signers (mean age $=37 \mathrm{yrs,}$ sd=9 yrs): 14 native, 14 early and 12 late.

\section{Results}

In LIS, no clear pattern emerged between forwards and backwards verbs, and native signers seemed to perform better than early and late signers in the mismatch condition only. In LSF, performance looked worse for backwards verbs in the match condition, and in general slightly better for native signers compared to the other groups.
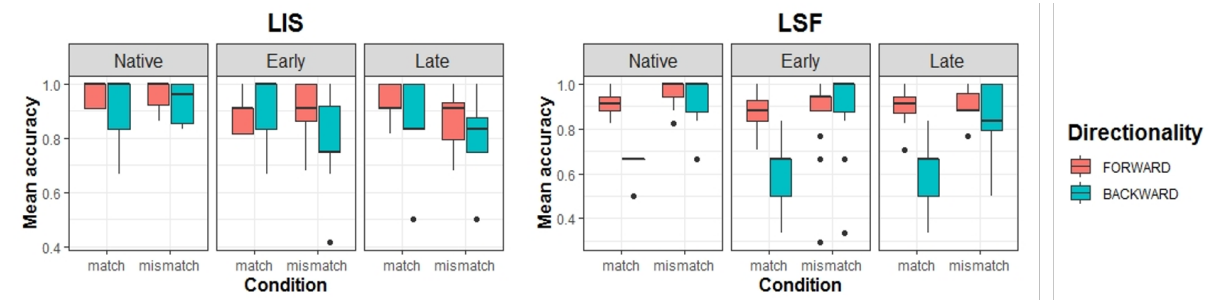

Figure 2. LIS and LSF results considering group, condition and verb directionality.

Analysis was performed separately for each SL, and for matching/mismatching sentences. We used generalized linear mixed models with random intercepts for subjects and items, and by-subject random slopes for the effect of directionality and by-item random slopes for the effect of group. Fixed factors were directionality, group, their interaction and participants' age. In LIS, native signers were more accurate in rejecting mismatching stimuli than early $(\beta=-1.83, \mathrm{SE}=0.54, \mathrm{z}=-3.41, \mathrm{p}<0.001)$ and late signers $(\beta=-1.94, \mathrm{SE}=0.54, \mathrm{z}=-3.66, \mathrm{p}<0.001)$. No significant difference was found comparing late and early signers. Verb directionality did not influence accuracy and no significant result was found in the match condition analysis. In LSF, native signers were more accurate than early and late signers in accepting matching and rejecting mismatched sentences (match - early: $\beta=-1.45$, $\mathrm{SE}=0.48, \quad \mathrm{z}=-3.05, \mathrm{p}=0.002$; late: $\beta=-1.11, \mathrm{SE}=0.47, \mathrm{z}=-2.37, \mathrm{p}=0.018$; mismatch - early: $\beta=-1.71, \mathrm{SE}=0.62, \mathrm{z}=-2.78, \mathrm{p}=0.005$; late: $\beta=-1.10$, $\mathrm{SE}=0.58, \quad \mathrm{z}=-1.88, \quad \mathrm{p}=0.06)$. In accepting matching sentences, verb directionality had a marginal impact $(\mathrm{p}=0.08)$. 


\section{Discussion and conclusion}

In both languages, we found that natives outperformed non-native signers. This result shows that the capacity of interpreting directionality in LIS and LSF is affected when access to language is delayed, coherently with previous results in other SLs. To us, this result is consistent with those approaches that take directionality to be a fully linguistic phenomenon, and can be interpreted as another evidence for the existence of a sensible period for language acquisition. As nothing similar to sensitive periods has been established (yet) for gestural systems, our findings do not support approaches that take directionality to be gestural. Moreover, our results highlight that delayed first language exposure has long lasting effects in adulthood. This finding, replicated across languages and linguistic structures, gives clear information for health-policy makers dealing with prelingual hearing loss.

Only in LSF we found a marginal effect of inverse directionality (backwards verbs were slightly more difficult than forwards verbs). This cross linguistic difference needs further investigation.

\section{Acknowledgements}

We are grateful to all participants who took part in the study. Many thanks to: Anna Folchi, Rosella Ottolini, Mirko Pasquotto, Mauro Mottinelli, Gabriele Caia, Antonino D'Urso for their contribution as LIS consultants; Valerie Jendoubi for her contribution as LSF consultant. This research was supported by the SIGN-HUB project (European Union's Horizon 2020 research and innovation program, Grant Agreement N 693349).

\section{References}

Cormier, K., Schembri, A., Vinson, D., Orfanidou, E. 2012. First language acquisition differs from second language acquisition in prelingually deaf signers: Evidence from sensitivity to grammaticality judgement in British Sign Language. Cognition 124, 50 65.

Emmorey, K., Bellugi, U., Friederici, A., Horn, P. 1995. Effects of age of acquisition on grammatical sensitivity: Evidence. Applied Psycholinguistics 16, 1-23.

Liddell, S. 1995. Real, surrogate, and token space: Grammatical consequences in ASL. In Emmorey, K., Reilly, L. (eds.) 1995, Language, gesture, and space, 19-41. Hillsdale NJ, Erlbaum.

Padden, C. A. (1998). The ASL lexicon. Sign language \& linguistics 1, 39-60.

Pfau, R., Salzmann, M., Steinbach, M. (2018). The syntax of sign language agreement: Common ingredients, but unusual recipe. Glossa: a journal of general linguistics 3 , 107. 